\title{
Antimicrobial Sensitivity Pattern of the Common Uropathogens among Patients Admitted in A Tertiary Care Hospital in Bangladesh
}

\author{
AKM Humayon Kabir ${ }^{1}$, SK Jakaria Been Sayeed ${ }^{2}$, Prodip Kumar Biswas ${ }^{3}$, SM Hafiz${ }^{4}$, Md. Uzzwal Mallik ${ }^{5}$ \\ Md. Nazim Uddin 6
}

\begin{abstract}
Urinary tract infection is one of the most common bacterial infections seen in clinical practice both in developed and developing countries. The causative agents of Urinary tract infection vary from place to place and they also vary in their susceptibility and resistance patterns. This descriptive study aimed to evaluate the antibiotic susceptibility patterns of pathogens isolated from routine laboratory specimens at Dhaka Medical College Hospital. A descriptive cross- sectional study was done from January 2018 to June 2018. A total of 100 mid-stream urine samples from the suspected UTI patients were tested microbiologically and antimicrobial susceptibility test were performed for the isolated pathogens using Kirby-Bauer disk diffusion method with positivity rate of UTI was 90\% (90/I00). Escherichia coli (39\%) was the most UTI causing bacteria followed by Klebsiella I8(I8\%), Acinetobacter 12(I2\%), Staphylococci, 9 (9\%), Pseudomonas 6 (6\%), Enterococcus species $3(3 \%)$ and Proteus $3(3 \%)$. Female 83 (83\%) were more affected in comparison with male. Adult female especially age range 18-30 years were found in high risk. Nitrofurantoin, Amikacin and Meropenem were recorded as most sensitive antibiogram for most of the bacteria's whereas cephalosporin showed increased resistance. However, for uncomplicated community acquired UTI, Nitrofurantoin (oral) and Amikacin (Injectable) can be chosen as first line medication.
\end{abstract}

Key words: Urinary Tract infection, Antibiogram, Etiological Pattern

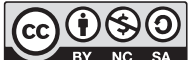

DOI: https://doi.org//0.3329/jom.v2 Ii2.502 I 2

Copyright: (C) 2020 Kabir AKMH et al. This is an open access article published under the Creative Commons Attribution-NonCommercial-NoDerivatives 4.0 International License, which permits use, distribution and reproduction in any medium, provided the original work is properly cited, is not changed in any way and it is not used for commercial purposes.

Received: 05 August 2020;

Accepted: II October 2020

\section{Introduction}

Urinary tract infections (UTI) is most common bacterial infections, affecting 150 million people each year worldwide, occurring from the neonate to geriatric age group. ${ }^{1}$ Nearly $30-40 \%$ of women will have had at least 1 episode of UTI

1. Associate Professor, Dept. of Medicine, Dhaka Medical College Hospital, Dhaka.

2. Indoor Medical Officer, Dept. of Medicine, Dhaka Medical College Hospital, Dhaka.

3. Associate Professor, Dept. of Medicine, Dhaka Medical College Hospital, Dhaka.

4. Professor of Medicine, Dept. of Medicine, Dhaka Medical College Hospital, Dhaka.

5. Assistant Professor, Dept. of Medicine, Dhaka Medical College Hospital, Dhaka.

6. Indoor Medical Officer, Dept. of Medicine, Dhaka Medical College Hospital, Dhaka.

Corresponding Author: Dr. A.K.M. Humayon Kabir, Associate Professor, Dept. of Medicine, Dhaka Medical College Hospital, Dhaka. E-mail: email-drakmhkabir@gmail.com. Cell no: $+8801711137528$ requiring antimicrobial therapy by the age of 24 years. Almost half of all women will experience one UTI during their lifetime, some $25 \%-50 \%$ experience recurrent episodes that result in repeated hospital visits, antimicrobial use and other morbidity and costs. ${ }^{2}$ In Bangladesh, $20 \%-35 \%$ females experience at least one episode of UTI in their lives where prevalence of bacteriuria among the pregnant women in Bangladesh has been reported from 5 to $15.5 \%{ }^{3,4}$ Urinary tract infection is a common contagion among men and women the prevalence is higher among sexually active women and during pregnancy 8 . Similarly there are some other factors that increase the risk of developing urinary tract infection e.g. age, minor urethral trauma during sexual intercourse especially in female, kidney stone, tumours, urethral strictures, enlarged prostate, vesico-ureteric reflex, neurological diseases, catheterization, diabetes mellitus, immunosuppression etc. ${ }^{2}$ Among the bacterial species Escherichia coli account to $80 \%$ to $85 \%$ of the infection followed by Staphylococcus species constitutes to $10 \%$ 
to $15 \%$. In addition, bacterial species Klebsiella, Pseudomonas, Proteus and Enterococcus species plays a minor role in conferring the infection. ${ }^{5}$ Emergences of resistant bacterial strains in hospitals pose a continued challenge to treat and control the spreading of infections. Moreover, the indiscriminate use of antibiotics often results in the increased resistance of urinary pathogens to most commonly used antimicrobial drugs causing a serious public health issue, particularly in the developing world. ${ }^{6}$ The successful management urinary tract infections \& prevention of recurrence depends upon the identification of the types of organisms, drug sensitivities and risk factors responsible for bacterial invasion and the selection of an effective antibiotic according to culture sensitivity. There is a paucity of research addressing the aetiologies, risk factors and pattern of antibiotic resistance of UTI patients in most developing countries. Therefore, this study was done to identify the type of organism(s) responsible and its sensitivity pattern in patients presented with pyuria and also to find out the associated risk factors that may complicate the condition.

\section{Methodology}

This was a descriptive type of cross-sectional study, conducted in inpatient department of medicine of Dhaka Medical College Hospital starting from January, 2018 to June, 2018. All patients suspected as UTI by physician were included for this study. Patients were informed about the study. Morning midstream urine samples were requested or preferred before antibiotic administration. For those patients who were already with antibiotics we instruct them to wait to void urine till the next dose of antibiotic. All the patients were clearly informed to clean the genital area with soap before collecting the midstream urine. All samples were processed to test within one hour of the collection. Antibiotic discs for sensitivity were used as per CSLI guidelines to check sensitive, resistant and intermediate antibiotics patterns, which were measured by antibiotic zones scale. Qualitative data are described as frequency or percentage and quantitative data as mean (SD) or median (interquartile range) as appropriate. Continuous and categorical data will be compared using Student's t-test and chi-square test respectively. Comparison will be done by tabulation and graphical presentation in the form of tables, bar diagrams and charts. All statistics will be performed using Statistical Package for the Social Sciences (SPSS) software. A p-value of $£ 0.05$ will be considered as statistically significant. Ethical clearance was taken from ethical review committee of respective institute.

\section{Results}

Out of 100 patients, $83(84 \%)$ were female and male to female ratio was $1: 4.88$. Female are more frequently affected in all age group from very young to aged. The age range was 18 to 90 years and mean age was $45 \pm 10.64$ years. Among 100 patients, 45 patients (45\%) belong to $18-30$ years followed by 18 (18\%) from 31-45 years group, 21 (21\%) in 46-60 years group whereas more than 60 years age group was $16 \%$ (Table 1). 92(92\%) patients were symptomatic and rest of them were asymptomatic. Most common risk factor associated with UTI were DM 36 (36\%) then menopause $18(18 \%)$, pregnancy $8(8 \%)$, catheter or stent $7(7 \%)$, renal stone $6(6 \%)$, prostate pathology $6(6 \%)$ etc. However, $10 \%$ remains unknown (Table 2 ). Majority (85\%) of patients had single risk factor, $5 \%$ had more than one and $10 \%$ had no risk factors at all. In bacterial strain analysis in urine most common pathogen found was E. coli 39(39\%), Klebsiella 18(18\%), Acinetobacter 12 (12\%), Staph aureus 9 (9\%), Pseudomonas $6(6 \%)$, Enterococcus species $3(3 \%)$ and Proteus $3(3 \%)$. However, in blood culture of 100 patient only 4 (4\%) had culture positive; those were E. coli (2) \& Staph. aureus (2) (Figure 1). Moreover, isolation of more than one organism found only in 5 patients $(5 \%)$.specially in diabetic \& catheter induced UTI cases. E-coli was highly sensitive to Amikacin (96\%), Meropenem (100\%), Nitrofurantoin (92\%) but for Ceftriaxone (72\%), Quinolone (62\%), Cefixime (69\%), sensitivity was low for Cephradine (38\%), Co-trimoxazole (38\%). However, Klebsiella was sensitive for Meropenem (100\%), Quinolone (83\%), Amikacin (83\%) but for Ceftriaxone it was only 50\%. $12(100 \%)$ of 12 patients with Acinetobacter were resistant to Ceftriaxone, Cefixime and Cephradine (Table 3). All were sensitive to Ceftazidime, Cefipime and Meropenem/ Imipenem. 9(75\%) patients were sensitive to Quinolone and Amikacin /gentamicin. Almost $9(100 \%)$ of 9 patients of Staphylococcus Aureus (MSSA) group were sensitive to Cloxacillin, Linezolid, Cephradine. All of the pseudomonas group were sensitive to Quinolone and Amikacin/gentamicin and also to Ceftazidime, Cefipime, Meropenem/Imipenem (Table 3)

Table I : Age \& Sex distribution of 100 patients presented with UTI

\begin{tabular}{lccc}
\hline $\begin{array}{l}\text { Age group } \\
\text { (years) }\end{array}$ & $\begin{array}{c}\text { Male } \\
(\mathrm{n}=17)\end{array}$ & $\begin{array}{c}\text { Female } \\
(\mathrm{n}=83)\end{array}$ & $\mathrm{N}(\%)$ \\
\hline $18-30$ & 4 & 41 & $45(45 \%)$ \\
$31-45$ & 2 & 16 & $18(18 \%)$ \\
$46-60$ & 5 & 16 & $21(21 \%)$ \\
$61-75$ & 4 & 8 & $12(12 \%)$ \\
$76-90$ & 2 & 2 & $4(4 \%)$ \\
\hline
\end{tabular}


Table II: Common risk factors found in 100 UTI patients

\begin{tabular}{lc}
\hline Risk factor & Frequency (\%) \\
\hline Diabetes & $36(36 \%)$ \\
Presence of Catheter(6) or stent (1) & $7(7 \%)$ \\
Renal stone disease & $6(6 \%)$ \\
Post-menopausal & $18(18 \%)$ \\
Pregnancy & $8(8 \%)$ \\
Prostatic enlargement & $6(6 \%)$ \\
Carcinoma prostate & $1(1 \%)$ \\
Neurological disorder & $2(2 \%)$ \\
History of previous UTI & $6(6 \%)$ \\
Unknown & $10(10 \%)$ \\
\hline
\end{tabular}

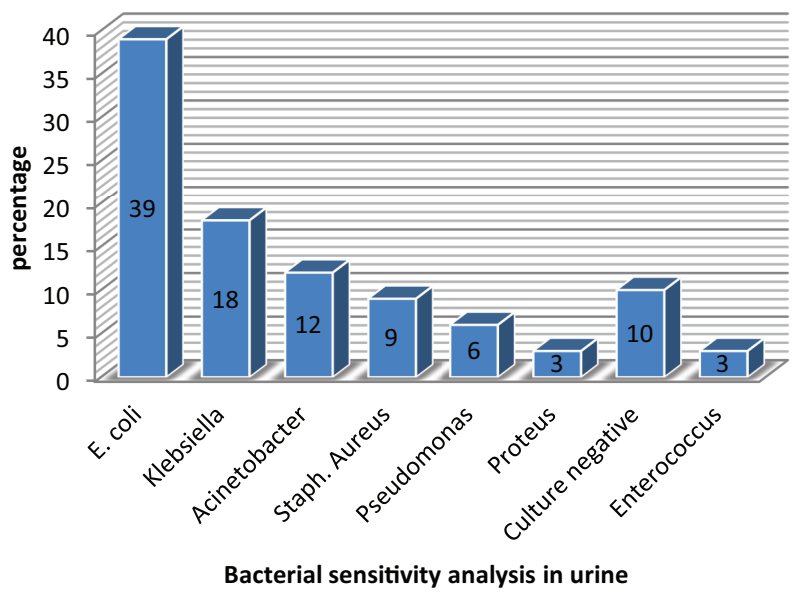

Fig.-1: Bacterial strain analysis from urine sample among 100 UTI patients

Table-III: Percentage of in vitro antibiotic sensitive pattern of most frequently isolated microorganisms

\begin{tabular}{lcccccc}
\hline Antibiotics & E. coli(\%) & Klebsiella (\%) & Acinetobacter (\%) & Staph. aureus (\%) & Pseudomonas (\%) & P value \\
\hline Nitrofurantoin & 92 & 67 & 100 & 75 & Not done & $\mathbf{0 . 0 2}$ \\
Amikacin & 100 & 83 & 75 & 75 & 83 & $\mathbf{0 . 0 1}$ \\
Ciprofloxacin & 62 & 83 & 75 & Not done & 83 & 0.28 \\
Co-trimoxazole & 38 & 83 & 75 & 50 & Resistant & 0.97 \\
Cephradine & 38 & Resistant & Resistant & 100 & Not done & 0.65 \\
Cefixime & 69 & 16 & Resistant & Not done & Not done & 0.21 \\
Ceftriaxone & 72 & 50 & Resistant & Not done & 50 & 0.43 \\
Ceftazidime & 92 & 72 & 100 & Not done & 83 & $\mathbf{0 . 0 4}$ \\
Meropenem & 100 & 100 & 100 & 100 & 100 & $\mathbf{0 . 0 0 0 1}$ \\
\hline
\end{tabular}

\section{Discussion}

The present study was undertaken on 100 consecutive patients of both sexes presented with pyuria. Among them $83(83 \%)$ were female. Male-female ratio was 1:4.88. A large study was conducted on 2013 at Dhaka city reveals 79\% female were suffering from culture positive UTI and remaining $21 \%$ was male. ${ }^{7}$ Several studies shows that UTIs are most commonly seen in sexually active young women. ${ }^{8}$ High prevalence of UTI among female population may be due to short and wide urethra and proximity to anus that makes easy for bacteria to ascend in the urinary tract and decrease of normal vaginal flora (Lactobacilli). Other factors that may contribute to develop UTI in women include urethral injury during sexual intercourse, diaphragmspermicide use, voiding behaviour \& poor hygienic conditions especially in low income society. ${ }^{7}$ Female may be affected with UTI at any age from reproductive age to elderly aged group. In my study population, the age range was 18 to 90 years and $63 \%$ of whom belong to women of reproductive age range from $18-45$ years. It is very similar to a study conducted in Bangladesh shown women has the age group 16 - 45 years are most likely to suffer from UTI. ${ }^{7}$ ${ }^{9}$ In our study, Diabetes was found in 36 patients (36\%) and many of them had have recurrent UTI, among them 17 had uncontrolled DM. A large study was conducted in Kuwait, where most of the UTI cases $(78.2 \%)$ were found in the diabetic patients with uncontrolled glycaemia. ${ }^{10}$ Moreover, we observed 8 cases $(8 \%)$ found to have pregnancy. Pregnancy is an independent risk factor of Urinary tract infection. In a study at Pakistan it shows 5\% cases were found where risk factor was pregnancy. ${ }^{8}$ Another study in Bangladesh show prevalence of UTI in pregnancy was $26 \%$. Individuals of the age group 21-25 years had the highest incidence of infection( $44.61 \%$ ) among them $76 \%$ infection 
occur at third trimester. ${ }^{11}$ However, present study showed low prevalence probably due to reduced admission in medicine ward. Here, 18 (18\%) postmenopausal women were suffering from UTI (Table 3). During menopause, the level of oestrogen decreases which cause changes in the tissues around the urethra and loss of protective vaginal flora and reduction of vaginal $\mathrm{P}^{\mathrm{H}}$ can lead to UTI. ${ }^{12}$ In this study, 6 UTI cases $(6 \%)$ was due to prostatic enlargement. In another study showed, $8-10 \%$ cases occur due to Prostate pathology. ${ }^{13,14}$

Most hospital-acquired UTIs are associated with urinary catheters, a commonly used device among hospitalized patients. Up to $25 \%$ of hospitalized patients have a urinary catheter placed during their stay. ${ }^{15,16}$ We found $6(6 \%)$ cases are due to catheter associated UTI that is $6(35 \%)$ out of 17 male patients suffering from UTI having urinary catheter. In this study, 5 patients had more than one risk factor like neurological disorder with CAUTI in 2 patients, postmenopausal status with diabetic complications in 3 patients. There is a large group $10 \%$ in where we could not find out any identifiable risk factor. A total of 7 types of bacterial pathogens were isolated from urine culture. Escherichia coli 39 (39\%) was the commonest bacterial pathogens in both symptomatic \& asymptomatic groups. Other pathogens were Klebsiella spp. 18(18\%) and most Acinetobacter spp. (12\%), Staph. aureus 9(9\%), Pseudomonas 6(6\%), Enterococcus species 3(3\%) and Proteus $3(3 \%)$ etc. So, gram negative bacilli were $88 \%$ of total uropathogens and remaining $12 \%$ were gram positive organisms included Staph. Aureus and Enterococcus spp. Akram M. et al. ${ }^{3}$ (2007) showed growth of pathogens among which the most prevalent were E. coli (61\%) followed by Klebsiella spp. (22\%). Forty two percent $(42 \%)$ of isolates were detected to produce ESBL among which $34.42 \%$ were E. coli isolates. Another study in North West India showed the showed that maximum patients were infected by Escherichia coli followed by Klebsiella, Staphylococci, Enterococci and Proteus species. ${ }^{17,18}$ However, two patients had both simultaneous infection in blood and urine. E. coli and Staph. aureus were causative organism. One of them was associated CAUTI and another one were associated with DJ stent in situ. Only 24(62\%) patients of 39 infected with Escherichia coli was sensitive to Quinolone group of antibiotic but $36(92 \%)$ patients were sensitive to Nitrofurantoin. $72 \%$ patients were sensitive to ceftriaxone, $69 \%$ to Cefixime but only $38 \%$ were sensitive to Cephradine as well as Co-trimoxazole. All were sensitive to Amikacin and Meropenem/Imipenem. 15(83\%) patients of 18 infected with Klebsiella spp. were sensitive to Quinolone group of antibiotic and also to Co-trimoxazole but only (83\%) patients were sensitive to Amikacin. All were sensitive to Meropenem/Imipenem. Only 50\% patients were sensitive to ceftriaxone but all were resistant to Cephradine. Very similar to a study from India, E. coli and Klebsiella were sensitive to Amikacin (97.1\%), Nitrofurantoin (90.7\%), Gentamycin and Imipenem. Both organisms were resistant to Ampicillin (> 70-90\%). ${ }^{19,20,21}$

\section{Limitations}

The study had the following limitations. The study period was short, sample size was small, no control group, single centred study. So it may not represent the scenario of whole country. These should be kept in mind while deciding on the implications of the findings of the study.

\section{Conclusion:}

Urinary tract infection is very common infection among the sexually active adult especially in women, can endanger life if neglected and if associated with anatomical risk factors and comorbidities. Antibiotic usage has proven value in encountering the infection. Identification of bacteria by culture sensitivity should be done in patient with nonresponsiveness to treatment and in case of recurrence, immunosuppression, pregnancy or complicated UTI. Escherichia coli remains the predominant uropathogen in acute community-acquired uncomplicated UTIs. Nitrofurantoin may be useful as a first-line oral antibiotic. If there is no contraindication, Amikacin can be first choice injectable antibiotic as most of the common uropathogens are sensitive to it. Identifying risk factor causing UTI is also important. Simple preventive measures may have important role in preventing recurrence of infection. We advise for a large scale multicentre study to evaluate the actual picture of sensitivity pattern of antibiotics in UTI.

\section{Acknowledgement}

Md. Helal Uddin, Medical Technologist, Popular diagnostic Centre, Dhaka.

\section{Conflict of Interest:}

The authors declare that there is no conflict of interests regarding the publication of this paper.

\section{References}

1. Martha Medina, Edgardo Castillo-Pino An introduction to the epidemiology and burden of urinary tract infections. Ther Adv Urol 2019, Vol. 11: 3-7.

2. Akram, M., Shahid, M. \& Khan, A.U. Etiology and antibiotic resistance patterns of community-acquired urinary tract infections in J N M C Hospital Aligarh, India. Ann Clin Microbiol 2007; Antimicrob 6, 4. https://doi.org/10.1186/ 1476-0711-6-4 
3. Ahmed S RH. Urinary tract infection in adults - a systematic review. Bangladesh Ren J 1996; 15(1):23-31.

4. Jhohra ST, Paul S, Begum B CAQ. Antibiogram of Ur inary E scherichia coli isolated in Sir Salimullah Medical College Mitford Hospital, Dhaka. Bangladesh J Med Microbiol 2011; 5(2):23-6.

5. Ronald A. The etiology of urinary tract infection: traditional and emerging pathogens. Am J Med. 2002;113( suppl 1A):14S-9S

6. Mansoor C. Abdulla, Feroz P. Jenner JA. Urinary tract infection in type 2 diabetic patients: risk factors and antimicrobial pattern. international J Res Med Sci 2015;3(10):2576-9.

7. L Paudel, N Manandhar, Saroj, S Khadka, S Neupane S. Prevalence of urinary tract infection and associated risk factors among women in Sindhupalchowk district, Nepal. Int J Community Med Public Heal 2018;5(7):2714-9.

8. M. Ramzan, S. Bakhsh, A. Salam, G. M. Khan GM. risk factors in urinary tract infection. Gomal J Med Sci [Internet] 2004;2(1):50-3.

9. Gupta S, Malakar M, Kalita P and Pandey K F. Bacterial profile and Antibiograms in urinary tract infection. Al Ameen J Med Sc i 2019; 12(4) : 192-196

10. Sewify M, Nair S, Warsame S, et al. Prevalence of Urinary Tract Infection and Antimicrobial Susceptibility among Diabetic Patients with Controlled and Uncontrolled Glycemia in Kuwait. J Diabetes Res. 2016; 2016:6573215. doi:10.1155/2016/6573215

11. Amala E GN. Prevalence of Asymtomatic Bacteriuria among Pregnant Women Attending Antenatal in Port Harcourt Township, Nigeria and Antibiogram of Isolated Bacteria. Am J Biomed Sci 2015;7(2):125-33

12. Raz R. Urinary tract infection in postmenopausal women. Korean J Urol. 2011; 52(12):801-808. doi:10.4111/ kju.2011.52.12.801

13. Nickel JC. Inflammatory and Pain Conditions of the Male Genitourinary Tract: Prostatitis and Related Pain Conditions,
Orchitis, and Epididymitis. Wein AJ, Kavoussi LR, Partin AW, Peters CA, eds. Campbell-Walsh Urology. 11th ed. Philadelphia, Pa: Elsevier; 2016. 304-33.

14. 9. M. Ramzan, S. Bakhsh, A. Salam, G. M. Khan GM. risk factors in urinary tract infection. Gomal J Med Sci [Internet] 2004;2(1):50-3.

15. Sanjay Saint, Christine P. Kowalski, Samuel R. Kaufman, Timothy P. Hofer, Carol A. Kauffman, Russell N. Olmsted, Jane Forman, Jane Banaszak-Holl, Laura Damschroder, Sarah L. Krein, Preventing Hospital-Acquired Urinary Tract Infection in the United States: A National Study, Clinical Infectious Diseases, Volume 46, Issue 2, 15 January 2008, Pages 243-250

16. Saint S, Wiese J, Amory Jk, et al. Are physicians aware of which of their patients have indwelling urinary catheters?, Am J Med, 2000, vol. 109 (pg. 476-80)

17. Sharma I, Laskar A, Paul D. Studies on Non-Cathethar related urinary tract infections from Assam, North-East India. Online International Interdisciplinary Research Journal, 2014; 4(2):88-94.

18. Gupta S, Malakar M, Kalita P and Pandey K F. Bacterial profile and Antibiograms in urinary tract infection. Al Ameen J Med Sc i 2019; 12(4) : 192-196

19. Jubina Bency A. T. Priyanka R., Ponnu Jose et al .A study on the bacteriological profile of urinary tract infection in adults and their antibiotic sensitivity pattern in a tertiary care hospital in central Kerala, India. Int J Res Med Sci. 2017 Feb;5(2):666-669 www.msjonline.org

20. Razak SK, Gurushantappa V. Bacteriology of urinary tract infection and antibiotic susceptibility pattern in a tertiary care hospital in South India. Int J Med Sci Public Health 2012; 1:109-12.

21. Chanda W, Manyepa M, Chikwanda E, Daka V, Chileshe J, Tembo M, et al. (2019) Evaluation of antibiotic susceptibility patterns of pathogens isolated from routine laboratory specimens at Ndola Teaching Hospital: A retrospective study. PLoS ONE 14(12): e0226676. https://doi.org/10.1371/ journal.pone. 0226676 . 